\title{
Vortex lattices in dipolar two-component Bose-Einstein condensates
}

\author{
N. Ghazanfari* \\ Department of Physics, Koç University, 34450 Istanbul, Turkey \\ and Department of Physics, Istanbul University, 34134 Istanbul, Turkey
}

A. Keleş

Department of Physics, University of Washington, Seattle, Washington 98195-1560, USA

M. Ö. Oktel

Department of Physics, Bilkent University, 06800 Ankara, Turkey

and Laboratory of Atomic and Solid State Physics, Cornell University, Ithaca, New York 14853-2501, USA

(Received 26 December 2013; published 21 February 2014)

\begin{abstract}
We consider a rapidly rotating two-component Bose-Einstein condensate with short-range $s$-wave interactions as well as dipolar coupling. We calculate the phase diagram of vortex lattice structures as a function of the intercomponent $s$-wave interaction and the strength of the dipolar interaction. We find that the long-range interactions cause new vortex lattice structures to be stable and lead to a richer phase diagram. Our results reduce to the previously found lattice structures for short-range interactions and single-component dipolar gases in the corresponding limits.
\end{abstract}

DOI: 10.1103/PhysRevA.89.025601

Cold-atom experiments provide the opportunity to study many-particle systems in a highly controlled manner. One of the novel regimes that have gained importance is the study of systems where the particles are interacting significantly through long-range dipolar forces [1-5]. The realization of the quantum degenerate gas of dipolar bosons and fermions [6-10] has given impetus to theoretical study of these systems in various parameter regimes [2-5].

The response of Bose-Einstein-condensed gases to rotation or an artificial magnetic field has been extensively investigated [11-20]. It has been well established that the ground state of a Bose-Einstein condensate (BEC) under rotation is a vortex lattice [11-19], and such lattices containing hundreds of vortices have been observed in experiments [21,22]. While the lattice structure for a single-component BEC with short-range interaction is always a triangular lattice, lattice structures of different symmetry can be obtained either by increasing the number of components in BECs or by introducing long-range interactions. The phase diagrams of the vortex lattice structures have been calculated for two-component [12] and spin-1 BECs [20]. Similarly, the effect of the long-range dipolar interactions on the vortex lattice structure of a single-component BEC has been investigated $[13,16,23]$. It is, thus, natural to ask how the long-range interactions modify the phase diagram of the two-component Bose condensates. In this Brief Report, we calculate the phase diagram of the vortex lattice structures as a function of both the $s$-wave interactions and the dipolar interactions. We determine the vortex lattice structures using the method developed in Ref. [12] for two-component condensates and generalized to dipolar interactions in Ref. [13].

We consider a disk-shaped rapidly rotating two-component Bose-Einstein condensate with contact and dipolar interactions. Each component can be considered a hyperfine state of the same atom. The orientation of the dipoles are assumed to

\footnotetext{
*nghazanfari@ku.edu.tr
}

PACS number(s): 67.85.Fg, 03.75.Lm

be fixed by the external field forming the trapping potential. The trap geometry is important in determining the nature of the interaction. For the disk-shaped condensates with the dipoles oriented along the symmetry axis, the interaction between atoms is predominantly repulsive. The extent of the cloud along the symmetry axis forms the effective cutoff for the short-range part of the dipolar interaction and can be utilized as a control over the dipolar forces. Similarly, the $s$-wave interaction strengths can be adjusted by Feshbach resonances, potentially creating a large phase space to explore. As our main aim is to understand the effects of long-range interactions on the vortex lattice structure of two-component condensates, we concentrate on a symmetric system where the two components have the same mass, the same density, and the same rotation frequency. The dipolar interactions are also assumed to be independent of the component. We calculate the equilibrium vortex lattice structures as a function of the strengths of the short-range intercomponent interaction and the component-independent dipolar interaction.

For a two-dimensional Bose-Einstein condensate confined in an isotropic harmonic trap with a frequency of $\omega$ and rotating at angular frequency $\Omega$ around the $z$ axis, the single-particle Hamiltonian is $H=\frac{P^{2}}{2 M}+\frac{1}{2} M \omega^{2} r^{2}-\Omega L_{z}$. Here $r^{2}=x^{2}+y^{2}, M$ is the mass of the particle, and $L_{z}$ is the total angular momentum in the $z$ direction. For such a system, $E_{m n}=\hbar(\omega+\Omega) n+\hbar(\omega-\Omega) m+\hbar \omega$ are the energy eigenvalues, and the corresponding eigenfunctions are $\phi_{n m} \propto$ $e^{r^{2} / 2 a^{2}}\left(\partial_{x}+i \partial_{y}\right)^{n}\left(\partial_{x}-i \partial_{y}\right)^{m}\left(e^{-r^{2} / a^{2}}\right)$, where $n \geqslant 0$ and $m \geqslant 0$, and $a=\sqrt{\frac{\hbar}{m \omega}}$. As shown in Ref. [11], when $\Omega$ is large enough, i.e., $\omega-\Omega$ is very small, the system fills the $n=0$ level or the lowest Landau level, known as the mean-field quantum Hall regime. The wave function in this regime, for an assembly of cold identical bosons rotating at frequency $\Omega$, can be written as a linear combination of single-particle eigenfunctions, $\Psi=f(z) e^{\frac{-r^{2}}{2 a}}$. Here $f(z)$ is an analytical function of $z=x+i y$. Thus, the zeros of $f$ are the positions of 
the vortices, which are assumed to form an infinite lattice. For a finite condensate, the vortex positions show small deviations from the regular lattice, resulting in a Thomas-Fermi density profile rather than a Gaussian [24]. As we are concerned with the changes in the structure of the lattice, we neglect these finite-size effects.

For a two-component Bose-Einstein condensate, each component is described with a condensate wave function $\Psi_{i}$, where $i=1,2$. The short-range $s$-wave interactions and the long-range dipole-dipole interactions are included in the energy functional

$$
\begin{aligned}
E[\Psi]= & \sum_{i=1,2} \int d^{2} \mathbf{r} \Psi_{i}^{*} H \Psi_{i}+\sum_{i, j=1,2} \frac{g_{i j}}{2} \int d^{2} \mathbf{r}\left|\Psi_{i}\right|^{2}\left|\Psi_{j}\right|^{2} \\
& +\sum_{i=1,2} \mu_{i}^{2} \int d^{2} \mathbf{r}_{1} d^{2} \mathbf{r}_{2}\left|\Psi_{i}\left(\mathbf{r}_{1}\right)\right|^{2} V\left(\mathbf{r}_{1}-\mathbf{r}_{2}\right)\left|\Psi_{i}\left(\mathbf{r}_{2}\right)\right|^{2} \\
& +\mu_{1} \mu_{2} \int d^{2} \mathbf{r}_{1} d^{2} \mathbf{r}_{2}\left|\Psi_{1}\left(\mathbf{r}_{1}\right)\right|^{2} V\left(\mathbf{r}_{1}-\mathbf{r}_{2}\right)\left|\Psi_{2}\left(\mathbf{r}_{2}\right)\right|^{2}
\end{aligned}
$$

where $g_{i i}=g_{i}=\frac{4 \pi \hbar^{2} a_{i}}{M}$ and $g_{12}=g_{21}=\frac{4 \pi \hbar^{2} a_{12}}{M}$ are the $s$ wave interaction constants between like and unlike atoms, respectively, and $\mu_{i}$ 's are the magnitudes of the magnetic dipole moment of each component. The magnetic dipoledipole interaction is $V\left(\mathbf{r}_{1}-\mathbf{r}_{2}\right)=\frac{\mu_{0}}{4 \pi} \frac{1}{\left|\mathbf{r}_{1}-\mathbf{r}_{2}\right|^{3}}$. Here we assume that the magnetic dipoles are parallel to each other, and perpendicular to the line joining the centers of the two dipoles. The densities of both components are considered to be equal. We assume that for the $s$-wave interactions $g_{1}=g_{2} \neq g_{12}$, and for the dipolar interactions $\mu_{1}=\mu_{2}=\mu$. The wave function of each component is normalized such that $\int d^{2} \mathbf{r}\left|\Psi_{i}\right|^{2}=N_{i}$. For a two-component Bose gas in which both components rotate with the same frequency, vortex lattices have the same structure, but one is shifted with respect to another. The wave functions for both components can be introduced by two basis vectors and one relative displacement vector. We assume that $\mathbf{B}_{1}$ and $\mathbf{B}_{2}$ are the basis vectors of the infinite lattice, and $\mathbf{r}_{0}=$ $c \mathbf{B}_{1}+d \mathbf{B}_{2}$ is the relative displacement of the vortex lattices in two components. The area of the unit cell is defined to be $v_{c}=$ $\left|\mathbf{B}_{1} \times \mathbf{B}_{2}\right|$. Since the condensate is in the mean-field quantum Hall regime, the density $|\Psi(\mathbf{r})|^{2}$ can be written as a product of a Gaussian and a function $n(\mathbf{r})$ which is periodic under lattice transformation $|\Psi(\mathbf{r})|^{2}=A e^{\frac{-r^{2}}{\sigma^{2}}} n(\mathbf{r})$ [11]. Here $\sigma$ is related to the number of the vortices and is given by $\frac{1}{\sigma^{2}}=\frac{1}{a^{2}}-\frac{\pi}{v_{c}}$. The periodic function $n(\mathbf{r})$ is expanded as $n(\mathbf{r})=\frac{1}{v_{c}} \sum_{\mathbf{K}} n_{\mathbf{K}} e^{i \mathbf{K} \cdot \mathbf{r}}$, where $\mathbf{K}_{i}$ 's are the reciprocal lattice vectors.

The presence of $|\Psi|^{4}$ and $\left|\Psi_{1}\right|^{2}\left|\Psi_{2}\right|^{2}$ in the energy functional leads us to define $I=\pi \sigma^{2} \int d^{2} \mathbf{r}\left|\Psi_{i}\right|^{4}$ and $I_{12}=\pi \sigma \int d^{2} \mathbf{r}\left|\Psi_{1}\right|^{2}\left|\Psi_{2}\right|^{2}$. In terms of Fourier coefficients, they are given as

$$
\begin{aligned}
I & =\sum_{\mathbf{K}, \mathbf{K}^{\prime}} \tilde{n}_{\mathbf{K}} \tilde{n}_{\mathbf{K}^{\prime}} e^{\frac{-\sigma^{2}\left|\mathbf{K}+\mathbf{K}^{\prime}\right|^{2}}{4}}, \\
I_{12} & =\sum_{\mathbf{K}, \mathbf{K}^{\prime}} \tilde{n}_{\mathbf{K}} \tilde{n}_{\mathbf{K}^{\prime}} e^{-i \mathbf{K} \cdot \mathbf{r}_{0}} e^{\frac{-\sigma^{2}\left|\mathbf{K}+\mathbf{K}^{\prime}\right|^{2}}{4}}, \\
\tilde{n}_{\mathbf{K}} & =\frac{n_{\mathbf{K}}}{\sum_{\mathbf{K}^{\prime}} n_{\mathbf{K}^{\prime}} e^{\frac{-\sigma^{2} \mathbf{K}^{\prime 2}}{4}}}
\end{aligned}
$$

In order to find the optimum vortex lattice structure, we express $n_{\mathbf{K}}$ 's in terms of the basis vectors. Introducing a complex representation for the basis vectors, $b_{i}=(\hat{\mathbf{x}}+i \hat{\mathbf{y}}) \cdot \mathbf{B}_{i}$ and choosing $\mathbf{B}_{1}$ to lay on the $x$ axis, the original basis vectors can be written as $\mathbf{B}_{1}=b_{1} \hat{\mathbf{x}}$ and $\mathbf{B}_{2}=b_{1}(u \hat{\mathbf{x}}+v \hat{\mathbf{y}})$, where $b_{2}=b_{1}(u+i v)$, and the area of the unit cell becomes $v_{c}=\left|\mathbf{B}_{1} \times \mathbf{B}_{2}\right|=b_{1}^{2} v$. The periodic part of the wave function can be chosen as the Jacobi $\Theta$ function [25], which has zeros forming a lattice, i.e., $f(z)=\Theta(\zeta, \tau) e^{\pi z^{2} / 2 v_{c}}$, where $\zeta=z / b_{1}$ and $\tau=b_{2} / b_{1}$. Fourier coefficients of $n_{\mathbf{K}}$ are easily calculated as $n_{\mathbf{K}}=(-1)^{m_{1}+m_{2}+m_{1} m_{2}} e^{\frac{-v_{c}|\mathbf{K}|^{2}}{8 \pi}} \sqrt{\frac{v_{c}}{2}}$, and $v_{c} \mathbf{K}^{2}=$ $\left(\frac{2 \pi}{v}\right)\left[\left(v m_{1}\right)^{2}+\left(m_{2}-u m_{1}\right)^{2}\right]$, for $\mathbf{K}=m_{1} \mathbf{K}_{1}+m_{2} \mathbf{K}_{2}$ with $\mathbf{K}_{1}$ and $\mathbf{K}_{2}$ the basis vectors of the reciprocal lattice $\left(\mathbf{K}_{1}=\frac{2 \pi}{v_{c}} \mathbf{B}_{2} \times \hat{\mathbf{z}}, \mathbf{K}_{2}=\frac{-2 \pi}{v_{c}} \mathbf{B}_{1} \times \hat{\mathbf{z}}\right)$, and $m_{1}$ and $m_{2}$ integers [12]. For a large number of vortices, the expression for $I$ and $I_{12}$ take the simple forms $I=\sum_{\mathbf{K}}\left|\frac{n_{\mathbf{K}}}{n_{0}}\right|^{2}$ and $I_{12}=\sum_{\mathbf{K}}\left|\frac{n_{\mathbf{K}}}{n_{0}}\right|^{2} \cos \mathbf{K} \cdot \mathbf{r}_{0}$. The $s$-wave interaction energy is

$$
E_{s}=\frac{g \rho^{2}}{\pi \sigma^{2}}\left(I+\frac{g_{12}}{g} I_{12}\right),
$$

with $\rho$ as the average density.

By following similar steps for the dipolar part of the energy expression, we write the dipole interaction energy in terms of the relative displacement, $r=r_{2}-r_{1}$, and the center of mass, $2 R=r_{1}+r_{2}$, coordinates, and then integrate with respect to $R$ to obtain

$$
E_{d}=\frac{\rho^{2} \mu_{0} \mu^{2}}{4 \pi \sigma^{2}} \sum_{\mathbf{K}}\left|\frac{n_{\mathbf{K}}}{n_{0}}\right|^{2}\left(1+\cos \mathbf{K} \cdot \mathbf{r}_{0}\right)\left[\frac{1}{\Lambda}-K\right]
$$

Here we define a cutoff, i.e., $\Lambda$, which is related to the thickness of the condensate, and regularize the system near the origin. In the limit of a large number of vortices the full interaction energy then can be written as

$$
E_{\mathrm{int}}=\frac{\rho^{2} \mu_{0} \mu^{2}}{4 \pi \sigma^{2} a}\left[\alpha I+\beta I_{12}-D\right],
$$

where $D=\sum_{\mathbf{K}}\left|\frac{n_{\mathbf{K}}}{n_{0}}\right|^{2} \mathbf{K} a\left(1+\cos \mathbf{K} \cdot \mathbf{r}_{0}\right), \alpha=\frac{4 g a}{\mu_{0} \mu^{2}}+\frac{a}{\Lambda}$, and $\beta=\frac{4 g_{12} a}{\mu_{0} \mu^{2}}+\frac{a}{\Lambda}$.

Since the dipole-dipole interaction is the same for like and unlike atoms, in the energy expression Eq. (7) $\alpha$ and $\beta$ can be interpreted as the energy contribution from the intracomponent and the intercomponent interactions, respectively. The coefficient $\alpha$ contains the short-range interaction parameter $g$ and it governs the internal behavior of the individual components. The coefficient $\beta$ contains intercomponent coupling and it determines the lattice offset between the two components. Because of the long-range nature of the dipolar interaction and its angular dependence, a cutoff is needed to regularize the interactions [13]. For the two-dimensional droplet harmonic trap considered here, the cutoff parameter $\Lambda$ can be taken as the width of the cloud in the narrow $(\hat{\mathbf{z}})$ direction.

We obtain the phase diagram of the system for different values of $\alpha$ and $\beta$ by minimizing the energy in Eq. (7) (see Fig. 1). We obtain seven different lattice structures as classified by their symmetry (see Fig. 2). In three of these phases, the vortices of both components appear at the same points; we call these structures the overlapped lattices. In 


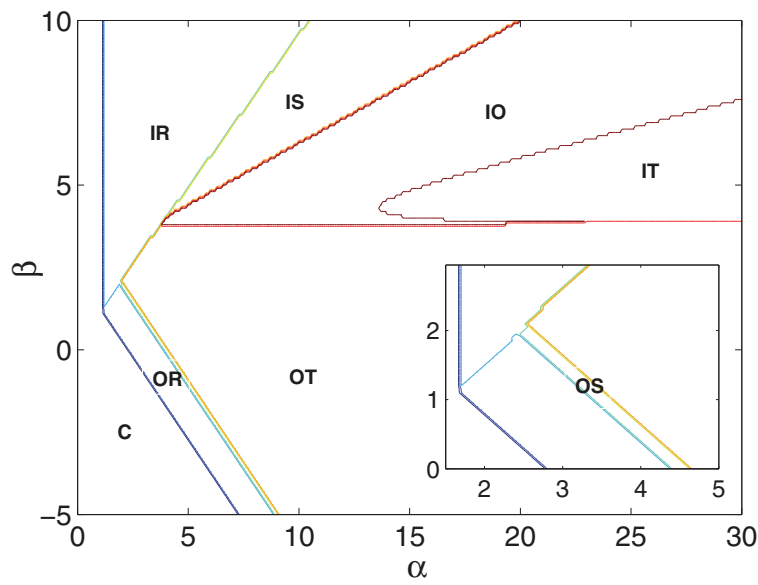

FIG. 1. (Color online) Phase diagram showing the lattice structures for different values of the interaction terms $\alpha$ and $\beta$. Here, $\mathrm{C}$ corresponds to the collapse region, and IR, IS, IO, IT, OR, OT, and OS stand for interlaced rectangular, interlaced square, interlaced oblique, interlaced triangle, overlapped rectangular, overlapped triangle, and overlapped square, respectively. The inset indicates the region for overlapped square lattices.

the remaining four phases the vortices of one component appear at the density maxima of the other component, creating interlaced lattices. As can be expected from our definition of $\beta$, the parameter controlling the intercomponent interaction, these two kinds of lattices are separated roughly by the line $\beta=3$. The dipolar interactions can cause the system to be

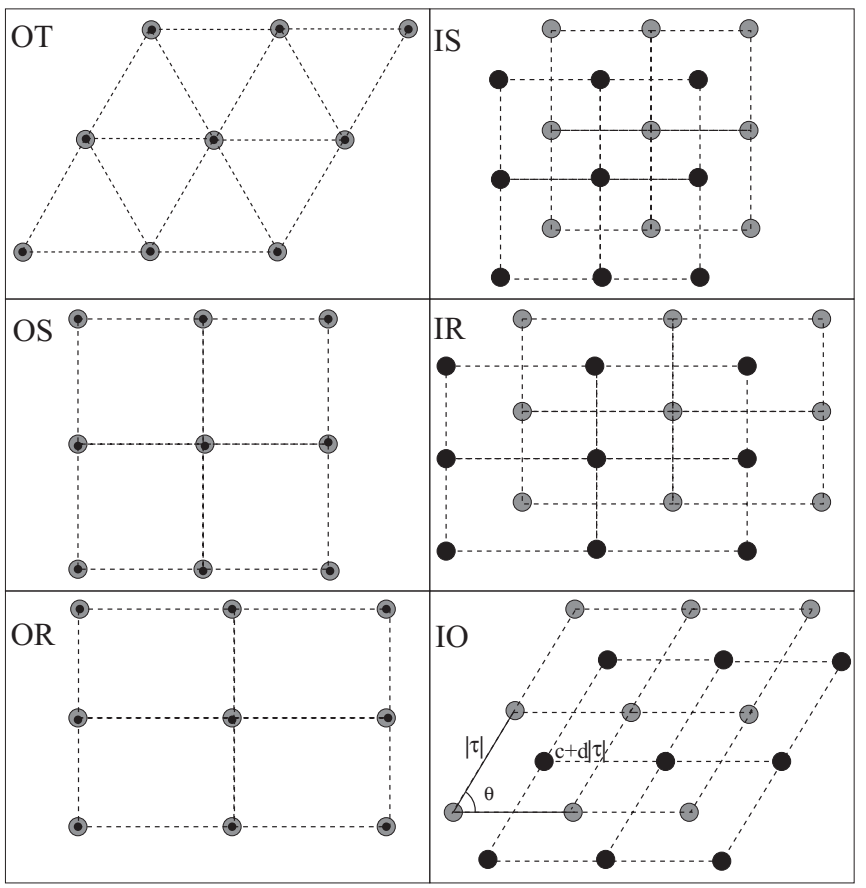

FIG. 2. Lattice structures for dipolar two-component condensates. Black and gray dots correspond to vortices in the two condensates. Lattice structures are defined in terms of aspect ratio $|\tau|$ and lattice angle $\theta ; c$ and $d$ determine the relative displacement between the two vortex lattices. The calculations are done assuming $c=d$. unstable, which we show as the collapse region in Fig. 1. While all four interlaced lattices have been found for the short-range-interacting systems, the overlapped rectangular and overlapped square lattices are stabilized in a gas with dipolar interactions.

The detailed analysis of the different aspects of the resulting phase diagram is given in the following points:

(i) The attractive interaction causes the condensate to collapse for $\alpha<1.25$. The condensate collapses even for large $\beta$ values, since it can not suppress internal fluctuations of each component.

(ii) For $\alpha>1.25$ and $\beta<1.25$, the intercomponent attraction is strong enough to overcome the dipolar repulsion between unlike atoms, which results in overlapped lattices. By increasing $\alpha$, the vortex structures undergo a structural phase transition from overlapped rectangular to overlapped square lattice and then to overlapped triangle lattices for higher $\alpha$ values.

(iii) For $1.25<\alpha<3.70$ and $1.25<\beta<3.70$, the ratio of these two parameters determines the relative displacement of the two lattices. In this region, when $\alpha<\beta$, an interlaced rectangular lattice is preferred. On the other hand, when $\alpha>\beta$, the minimum energy configuration is an overlapped triangular lattice.

(iv) When $\alpha \geqslant 3.70$ and $\beta \geqslant 3.70$, only interlaced lattices exist in the phase diagram, since the intercomponent interaction is not attractive in this regime. The repulsive forces between two different species cause the density minima of one component to move to the density maxima of the other component. In this region, upon increasing $\alpha$ the structure of the lattice changes from interlaced rectangular to interlaced square, oblique, and finally interlaced triangular.

Adjusting the strength of the parameters $\alpha$ and $\beta$ also enables us to control the switching between the regime of dominantly dipolar condensates and the regime of ordinary two-component condensates. One can easily conclude from Eq. (7) that for small values of $\alpha$ and $\beta$, the dipole-dipole interaction is dominant, and for large values of $\alpha$ and $\beta$, the contact interaction is more prominent. For large $\alpha$ and $\beta$, the last term $D$ can be ignored and lattice structures are determined by the ratio $\alpha / \beta$. Thus, the work reduces to the minimization of the term $J=I+\beta / \alpha I_{12}$. In the case of a dipole-dominant regime, when $g=g_{12}$, a two-component gas behaves like a single-component gas. Thus, the problem reduces exactly to the system studied in Ref. [13] as $E_{\text {int }}=\frac{n^{2} \mu_{0} \mu^{2}}{2 \pi \sigma^{2} a}[\alpha I-D]$.

To demonstrate the correspondence with Refs. [12] and [13], the phase diagrams along two different lines on the $\alpha \beta$ plane are shown in Fig. 3. Figure 3(a) gives the phase diagram for fixed $\alpha$ and changing $\beta$ that corresponds to two-component condensate with only the short-range interactions as in [12]. Figure 3(b) shows the phase diagram along an $\alpha=\beta$ line which corresponds to a single-component condensate with the short-range and dipolar interactions as in Ref. [13].

For $\alpha=\beta$, we observe the same vortex lattices obtained for the single-component Bose gas with dipolar interactions [see Fig. 3(b)]. However, the correspondence is not straightforward and requires careful examination:

(a) In the region $\alpha>4.54$, the triangular vortex lattice is observed when the two components are considered together. The individual components separately form rectangular 
(a)
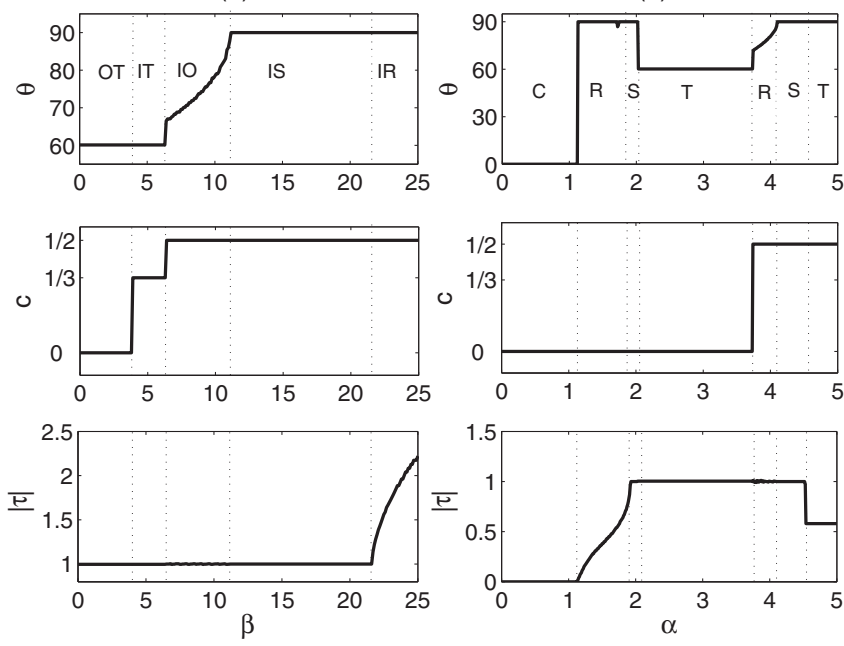

FIG. 3. The parameters indicating the type of lattice structures as a function of interaction coefficients $\alpha$ and $\beta$. (a) The limit for an ordinary two-component condensate, where $\alpha=20$. (b) The limit for a dipolar single-component condensate, i.e., $\alpha=\beta$. Here $|\tau|$ and $\theta$ are the lattice parameters and $c$ indicates the displacement of the lattice structure of one species with respect to the other.

lattices but they are interlaced such that the combined lattice is triangular. It is easy to observe this in Fig. 2 for IR (interlaced rectangular) lattices.

(b) In the region $4.1<\alpha<4.54$, the vortices of the two-component condensate form interlaced square lattices, but the combined lattice is again square with a smaller lattice constant.

(c) In the region $3.73<\alpha<4.1$, the two-component gas forms interlaced oblique lattices but the combined lattice is rectangular, regardless of the angle of the oblique lattices.

(d) In the region $1.12<\alpha<3.73$, the two components form overlapped lattices which both are identical to the combined lattice. The combined lattice goes through structural phase transitions in accordance with Ref. [13].

(e) In the region $\alpha<1.12$, the condensate collapses.

Compared to the short-range-interacting gas, two new lattice structures, overlapped square and overlapped rectangular, are stabilized as a result of dipolar interactions. As the correspondence to the single-component dipolar gas reveals, these structures are preferred as they maximize the attractive interaction at higher Fourier components of the real space density. A two-component gas with short-range interactions can take advantage of the fourfold rotational symmetry only when the two components repel each other, while the longrange dipolar interaction stabilizes the square lattice even for a single-component gas. The phase diagram shows that these two paths of lowering the energy barrier to fourfold symmetry are not mutually exclusive and square vortex lattices can exist for which the two components are locally attractive. Still, the overlapped square phase is very fragile and covers a relatively small area in the phase diagram.

In this Brief Report, we calculated the phase diagram of vortex lattice structures for a two-component BEC in the presence of dipolar interactions. Our results reduce to the ordinary two-component and dipolar single-component vortex lattices in the appropriate limits. Two more lattice structures, the overlapped square and overlapped rectangular lattices, are obtained as a result of dipolar interactions. Experimental observation of these two lattice types would be a clear indication of dominant dipolar interactions in a cold atomic gas.

N.G. is supported by TÜBITAK. A.K. is supported by the U.S. Department of Energy through Grant No. DE-FG0207ER46452. M.Ö.O. is supported by TÜBITAK Grant No. 112T974. M.Ö.O. thanks Cornell University for hospitality and the American Physical Society International Travel Award Grant for support. Part of this research was carried out at the Aspen Center for Physics, with the support of the Simons Foundation and NSF Grant No. 1066293.
[1] M. A. Baranov, K. Osterloh, and M. Lewenstein, Phys. Rev. Lett. 94, 070404 (2005).

[2] Uwe R. Fischer, Phys. Rev. A 73, 031602(R) (2006).

[3] S. Ronen, D. C. E. Bortolotti, and J. L. Bohn, Phys. Rev. Lett. 98, 030406 (2007).

[4] R. M. Wilson, S. Ronen, J. L. Bohn, and H. Pu, Phys. Rev. Lett. 100, 245302 (2008).

[5] N. G. Parker, C. Ticknor, A. M. Martin, and D. H. J. O’Dell, Phys. Rev. A 79, 013617 (2009).

[6] A. Griesmaier, J. Werner, S. Hensler, J. Stuhler, and T. Pfau, Phys. Rev. Lett. 94, 160401 (2005).

[7] T. Koch, T. Lahaye, J. Metz, B. Frohlich, A. Griesmaier, and T. Pfau, Nat. Phys. 4, 218 (2008).

[8] M. Lu, N. Q. Burdick, S. H. Youn, and B. L. Lev, Phys. Rev. Lett. 107, 190401 (2011).

[9] K. Aikawa, A. Frisch, M. Mark, S. Baier, A. Rietzler, R. Grimm, and F. Ferlaino, Phys. Rev. Lett. 108, 210401 (2012).
[10] M. Lu, N. Q. Burdick, and B. L. Lev, Phys. Rev. Lett. 108, 215301 (2012).

[11] T. L. Ho, Phys. Rev. Lett. 87, 060403 (2001).

[12] E. J. Mueller and T. L. Ho, Phys. Rev. Lett. 88, 180403 (2002).

[13] J. Zhang and H. Zhai, Phys. Rev. Lett. 95, 200403 (2005).

[14] M. O. Oktel, Phys. Rev. A 69, 023618 (2004).

[15] H. Zhai, Q. Zhou, R. Lu, and L. Chang, Phys. Rev. A 69, 063609 (2004).

[16] N. R. Cooper, E. H. Rezayi, and S. H. Simon, Phys. Rev. Lett. 95, 200402 (2005).

[17] R. Barnett, S. Mukerjee, and J. E. Moore, Phys. Rev. Lett. 100, 240405 (2008).

[18] P. Kuopanportti, J. A. M. Huhtamaki, and M. Mottonen, Phys. Rev. A 85, 043613 (2012).

[19] M. Cipriani and M. Nitta, Phys. Rev. Lett. 111, 170401 (2013). 
[20] T. Kita, T. Mizushima, and K. Machida, Phys. Rev. A 66, 061601 (2002).

[21] J. R. Abo-Shaeer, C. Raman, J. M. Vogels, and W. Ketterle, Science 292, 476 (2001).

[22] P. C. Haljan, I. Coddington, P. Engels, and E. A. Cornell, Phys. Rev. Lett. 87, 210403 (2001).
[23] S. Komineas and N. R. Cooper, Phys. Rev. A 75, 023623 (2007).

[24] V. Schweikhard, I. Coddington, P. Engels, V. P. Mogendorff, and E. A. Cornell, Phys. Rev. Lett. 92, 040404 (2004).

[25] M. Abramowitz and I. A. Stegun, Handbook of Mathematical Functions (Dover, New York, 1966). 